\title{
DURÉE MINIMALE DE PÊCHE POUR ESTIMER LE RENDEMENT DE PÊCHE DANS LES ENQUÊTES ITINÉRANTES AUPRÈS DES PÊCHEURS AUX LIGNES.
}

\author{
T. CHANGEUX (1), O. GALLET (2)
}

(1) Conseil Supérieur de la Pêche, Direction Générale, 134 avenue de Malakoff, F-75116 PARIS, France.

(2) Conseil Supérieur de la Pêche, Cellule Nationale Expérimentation des Méthodes de Gestion, 84 rue de Rennes, F-35510 CESSON SÉVIGNÉ, France.

\section{RÉSUMÉ}

Les enquêtes itinérantes auprès des pêcheurs aux lignes fournissent des indications sur des sessions de pêche incomplètes. Dans ce cas, il est recommandé d'estimer le rendement de pêche en effectuant la moyenne des ratios (captures divisées par temps de pêche), calculés pour chaque session de pêche après avoir éliminé les sessions les plus courtes.

La durée de pêche minimale pour retenir une session varie suivant la valeur du paramètre à estimer et la précision requise (fixée arbitrairement au niveau d'un coefficient de variation de $30 \%$ ). Les captures par unité d'effort de truite fario Salmo trutta, sur six parcours à salmonidés répartis à travers la France, sont en moyenne de 1,6 poisson/h. La durée minimale préconisée pour cette espèce est de $15 \mathrm{~min}$. Le même paramètre estimé pour le sandre Stizostedion lucioperca et le brochet Esox lucius, sur huit parcours répartis à travers la France, prend respectivement la valeur de 0,040 poisson $/ \mathrm{h}$ et 0,027 poisson $/ \mathrm{h}$. La durée minimale préconisée pour ces deux espèces est de $1 \mathrm{~h}$.

Mots-clés : enquête itinérante, pêcheurs aux lignes, estimation du rendement de pêche, durée de pêche minimale.

\section{MINIMUM FISHING SESSION DURATION FOR CATCH RATE ESTIMATION IN ROVING CREEL ANGLER SURVEYS.}

\begin{abstract}
Roving creel angler surveys give information on incomplete fishing sessions. In this case, it is recommended to estimate catch rate by using the mean of ratios (captures divided by time of fishing), calculated for each session excluding short sessions.

The minimum fishing duration necessary to include a session varies according to the value of the estimate and the admitted precision level (arbitrarily fixed to a coefficient of
\end{abstract}


variation equal to $30 \%$ ). The Brown Trout Salmo trutta capture per unit of effort, calculated across six different sectors distributed among France, have a mean value of $1.6 \mathrm{fish} / \mathrm{h}$. The recommended minimum duration is 15 minutes for this species. The same parameter for Pikeperch Stizostedion lucioperca and Pike Esox lucius, calculated across eight different sectors distributed among France, is respectively $0.040 \mathrm{fish} / \mathrm{h}$ and $0.027 \mathrm{fish} / \mathrm{h}$. The recommended minimum duration for the two species is 1 hour. duration.

Key-words : roving creel survey, angler, catch rate estimation, minimum session

\section{INTRODUCTION}

Le rendement de pêche est utilisé principalement dans trois objectifs : (1) estimer les captures totales d'une pêcherie pendant un temps donné, (2) obtenir un indice de densité du stock, (3) mesurer la qualité et le succès de pêche (MALVESTUTO, 1996 ; GERARD, 1993).

Pour les pêcheurs aux lignes, la moyenne de ce paramètre peut être estimée de deux manières différentes :

- en faisant la moyenne des ratios :

$$
\hat{R}_{1}=\frac{\sum_{i=1}^{n} \frac{c_{i}}{e_{i}}}{n}
$$

- en faisant le ratio des moyennes (ou des sommes ce qui revient au même) :

$$
\mathrm{R}_{2}=\frac{\frac{\left(\sum_{i=1}^{n} c_{i}\right)}{n}}{\frac{\left(\sum_{i=1}^{n} e_{i}\right)}{n}}=\frac{\sum_{i=1}^{n} c_{i}}{\sum_{i=1}^{n} e_{i}}
$$

où $c_{i}$ est le nombre de captures, $e_{i}$ le nombre d'heures de pêche de la session de pêche $i$, et $n$ le nombre total de sessions prises en comptes.

Les données sont recueillies auprès des pêcheurs, soit par carnets de pêche, soit par enquête. Dans les calculs, on distingue d'une part les sessions de pêche complètes, obtenues au travers de carnets de pêche ou d'enquêtes au point d'accès (« acces point surveys »), et d'autre part les sessions de pêche incomplètes, obtenues par enquêtes itinérantes (« roving creel surveys ») en interrompant le pêcheur pendant son action de pêche. 
Le choix du meilleur estimateur est resté longtemps controversé, d'autant plus que les résultats obtenus sont souvent très différents quelle que soit la taille de l'échantillon (POLLOCK et al., 1994, p. 179). Lorsque les calculs sont faits à partir d'enquêtes itinérantes, donc de sessions de pêche incomplètes, il est recommandé d'utiliser la moyenne des ratios sans tenir compte des sessions de pêche les plus courtes (HOENIG et al., 1997 ; POLLOCK et al., 1997). En effet, théoriquement lorsqu'on divise les captures par un temps de pêche proche de zéro, le ratio tend vers l'infini. En écartant les temps de pêche les plus faibles, on stabilise la variance de l'estimateur.

HOENIG et al. (1997) et POLLOCK et al. (1997) recommandent d'éliminer les sessions de pêche inférieures à 30 minutes. Ils s'appuient sur plusieurs simulations utilisant un rendement de pêche distribué selon une loi de Poisson de moyenne 2 poisson/h. Mais la durée minimale de pêche à prendre en compte dépend également de la probabilité de capture qui varie avec la densité du stock et la capturabilité des poissons.

Le présent article a pour objectif de déterminer, à partir de données réelles, les durées minimales qu'il est souhaitable de retenir pour trois espèces plus particulièrement recherchées par les pêcheurs aux lignes français : la truite fario Salmo trutta, le sandre Stizostedion lucioperca, et le brochet Esox lucius.

\section{MATÉRIEL ET MÉTHODES}

\section{Données utilisées}

Deux jeux de données sont utilisés. Ils proviennent d'enquêtes itinérantes réalisées depuis 1997 par les brigades du Conseil Supérieur de la Pêche à travers toute la France (GALLET, 2001). L'enquêteur demande au pêcheur l'espèce recherchée, son temps de pêche à 15 minutes près, et note le nombre de poissons capturés par espèce. II s'agit de capture par unité d'effort (CPUE), car même les poissons remis à l'eau sont pris en compte.

Le premier jeu de données comprend 875 sessions de pêche consacrées à la recherche de la truite fario sur six parcours à salmonidés : Ster-goz dans le Finistère, de 1998 à 2001 ; Albarine dans l'Ain en 2000 ; Pique, Job et Arbas en Haute-Garonne en 2000 ; Garbet en Ariège en 2000. Le temps de pêche varie de 15 minutes à 8 heures.

Le deuxième jeu de données comprend respectivement 2137 et 1983 sessions de pêche consacrées à la recherche du brochet et/ou du sandre sur huit parcours à cyprinidés : Cébron dans les Deux-Sèvres de 1999 à 2000 ; Vilaine dans l'llle-et-Vilaine de 1997 à 2001 ; Etang Boulet dans l'llle et Vilaine de 1997 à 1999 ; Couesnon dans les Côtes-d'Armor de 1997 à 1999, Vienne et Cher dans l'Indre-et-Loire en 2000, marais de l'Aure et de la Dive dans le Calvados en 2000. Le temps de pêche varie de 15 minutes à 11 heures.

Ces parcours se situent dans des unités de gestion (" contextes " au sens de CHANGEUX et al., 2001) dont l'état écologique s'éloigne quelque peu de la situation observée à l'échelle de la France (Tableau I). Les contextes salmonicoles conformes sont proportionnellement plus nombreux dans notre échantillon qu'à l'échelon national. Les contextes cyprinicoles dégradés sont également mieux représentés dans notre échantillon qu'à l'échelon national. 


\begin{tabular}{|c|c|c|c|c|c|c|}
\hline $\begin{array}{c}\text { Etat } \\
\text { écologique }\end{array}$ & $\begin{array}{l}\text { Contextes } \\
\text { salmonicole }\end{array}$ & $\begin{array}{c}\text { Echantillon } \\
(\%)\end{array}$ & $\begin{array}{c}\text { National } \\
(\%)\end{array}$ & $\begin{array}{l}\text { Contextes } \\
\text { cyprinicole }\end{array}$ & $\begin{array}{c}\text { Echantillon } \\
(\%)\end{array}$ & $\begin{array}{c}\text { National } \\
(\%)\end{array}$ \\
\hline Conforme & $\begin{array}{c}\text { Ster-goz, } \\
\text { Albarine, Job, } \\
\text { Garbet }\end{array}$ & $67 \%$ & $27 \%$ & & $0 \%$ & $10 \%$ \\
\hline Perturbé & Pique, Arbas & $33 \%$ & $54 \%$ & $\begin{array}{c}\text { Etang du Boulet, } \\
\text { Vienne, Marais } \\
\text { de l'Aure, Marais } \\
\text { de la Dive }\end{array}$ & $50 \%$ & $56 \%$ \\
\hline Dégradé & & $0 \%$ & $19 \%$ & $\begin{array}{l}\text { Cébron, Vilaine, } \\
\text { Couesnon, Cher }\end{array}$ & $50 \%$ & $34 \%$ \\
\hline Total & & $100 \%$ & $100 \%$ & & $100 \%$ & $100 \%$ \\
\hline
\end{tabular}

\section{Tableau I}

Répartition des parcours étudiés selon l'état écologique et le type (salmonicole ou cyprinicole) de l'unité de gestion (contexte) à laquelle ils se rapportent. Comparaison des proportions dans l'échantillon et au niveau national (selon NIHOUARN et CHANGEUX, données non publiées).

\section{Table I}

Distribution of the fishing areas according to ecological status and type (salmonid or cyprinid) of the management unit (context) they belong to. Comparison between occurrences in the sample and at the national level (from NIHOUARN et CHANGEUX, unpublished data).

\section{Méthode}

Les ratios élémentaires $c_{/} / e_{i}$ sont calculés pour chaque session de pêche $i$ avec $c$ nombre de poissons et $e_{i}$ temps de pêche. Ils sont établis pour le brochet et le sandre à partir du même jeu de données en tenant compte de l'espèce recherchée désignée par le pêcheur. Souvent le pêcheur indique rechercher les deux espèces à la fois. Sa session est alors prise en compte dans les deux calculs.

Pour connaître la précision de l'estimateur, nous avons étudié sa dispersion au travers de son erreur standard (SE), de son indice de confiance à $95 \%$ (IC) et de son coefficient de variation $(\mathrm{CV})$. Ces trois paramètres ont été établis à partir des indications de DAGNELI (1992) de la manière suivante :

$$
\begin{gathered}
S E\left(R_{1}\right)=\sqrt{\frac{n \sum_{i=1}^{n}\left(\frac{C_{i}}{e_{i}}\right)^{2}-\left(\sum_{i=1}^{n} \frac{C_{i}}{e_{i}}\right)^{2}}{n^{2}(n-1)}} \\
\operatorname{IC}\left(R_{1}\right)=1,96 \cdot \operatorname{SE}\left(R_{1}\right) \\
\operatorname{CV}\left(R_{1}\right)=\frac{S E\left(R_{1}\right)}{R_{1}}
\end{gathered}
$$


Pour mesurer l'influence des petits temps de pêche, les ratios élémentaires sont classés par durée de pêche croissante. Puis, ils sont intégrés progressivement dans les calculs, par groupe de 30 sessions de pêche minimum, en commençant par les valeurs les plus faibles de temps de pêche. Les groupes sont constitués en rassemblant les sessions avec un pas de temps de 15 minutes pour la truite et de 1 heure pour les carnassiers (brochet et sandre). Cette distinction de traitement prend en compte les biais d'arrondi et de souvenir décrits par POLLOCK et al. (1994, p. 71) qui touchent particulièrement les réponses apportées par les pêcheurs de carnassiers car ils ont des durées de pêche plus importantes.

\section{RÉSULTATS}

Les valeurs de l'estimateur avec son intervalle de confiance à $95 \%$ et son coefficient de variation sont données pour chaque espèce en fonction du nombre des sessions prises en compte (Figures 1, 2 et 3 ).

La truite (Figure 1) présente une valeur de CPUE dont la moyenne converge vers 1,62 poisson/h. En se limitant aux sessions de durées de pêche inférieures à 15 minutes, la moyenne est seulement de 1,26 poisson/h, l'intervalle de confiance à $95 \%$ représente $\pm 80 \%$ de part et d'autre de cette valeur, et le coefficient de variation s'élève à $41 \%$. Une fois étendu le calcul aux sessions de pêche de durée atteignant 30 minutes, la valeur de l'estimateur se rapproche de la valeur de convergence, le coefficient de variation est divisé par deux (19\% contre $41 \%)$ et l'intervalle de confiance à $95 \%$ également $( \pm 38 \%$ contre $\pm 80 \%$ ). Le domaine de variation diminue ensuite bien plus faiblement.

Le sandre (Figure 2) laisse apparaître un profil similaire à celui de la truite mais la valeur de convergence des CPUE est nettement plus faible $(0,040$ poisson $/ \mathrm{h}$ soit 1 poisson toute les $25 \mathrm{~h}$ ). La valeur de l'estimateur, calculée avec les sessions de durées inférieures à $1 \mathrm{~h}$, est assez proche de la valeur de convergence. Le coefficient de variation chute de $70 \%$ à $27 \%$ lorsqu'on étend la durée prise en compte au-delà de $1 \mathrm{~h}$ mais l'intervalle de confiance à $95 \%$ reste important $( \pm 53 \%)$.

Le brochet (Figure 3) présente des CPUE qui convergent vers une valeur légèrement plus faible que le sandre $(0,027$ poisson/h soit 1 poisson toutes les $37 \mathrm{~h})$. La valeur de l'estimateur, calculée avec les sessions de durées inférieures à $1 \mathrm{~h}$, est supérieure à la valeur de convergence. Le coefficient de variation passe de $50 \%$ à $28 \%$ lorsqu'on étend la durée prise en compte au-delà de $1 \mathrm{~h}$, mais l'intervalle de confiance à $95 \%$ reste important (56\%), comme pour le sandre.

\section{DISCUSSION}

\section{Les valeurs moyennes}

La valeur moyenne de CPUE truite est comparable aux 2 poisson/h utilisés par HOENIG et al. (1997) et POLLOCK et al. (1997) dans leurs simulations. Notre chiffre se situe dans les valeurs les plus élevées rapportées par FATORA (1974) entre 1 et 1,7 poisson/h. En revanche, les valeurs moyennes de CPUE brochet et sandre sont nettement inférieures. La moyenne de CPUE brochet est proche des 0,027 poisson $/ \mathrm{h}$ obtenus par KEMPINGER et CARLINE (1978), mais très inférieure aux valeurs de MACKENZIE (1991), situées entre 0,34 et 0,53 poisson/h. LATTA (1972) rapporte également des valeurs comparables aux nôtres, mais pour les seuls poissons supérieurs à la taille légale. Enfin, les CPUE de sandre peuvent être comparées aux résultats de HANSEN et al. (2000) sur le vitré (Stizostedion vitreum) : pour une densité moyenne de 2,8 poisson/acre la CPUE $=0,021(2,8)^{1,038}=0,06$ poisson $/ \mathrm{h}$. 


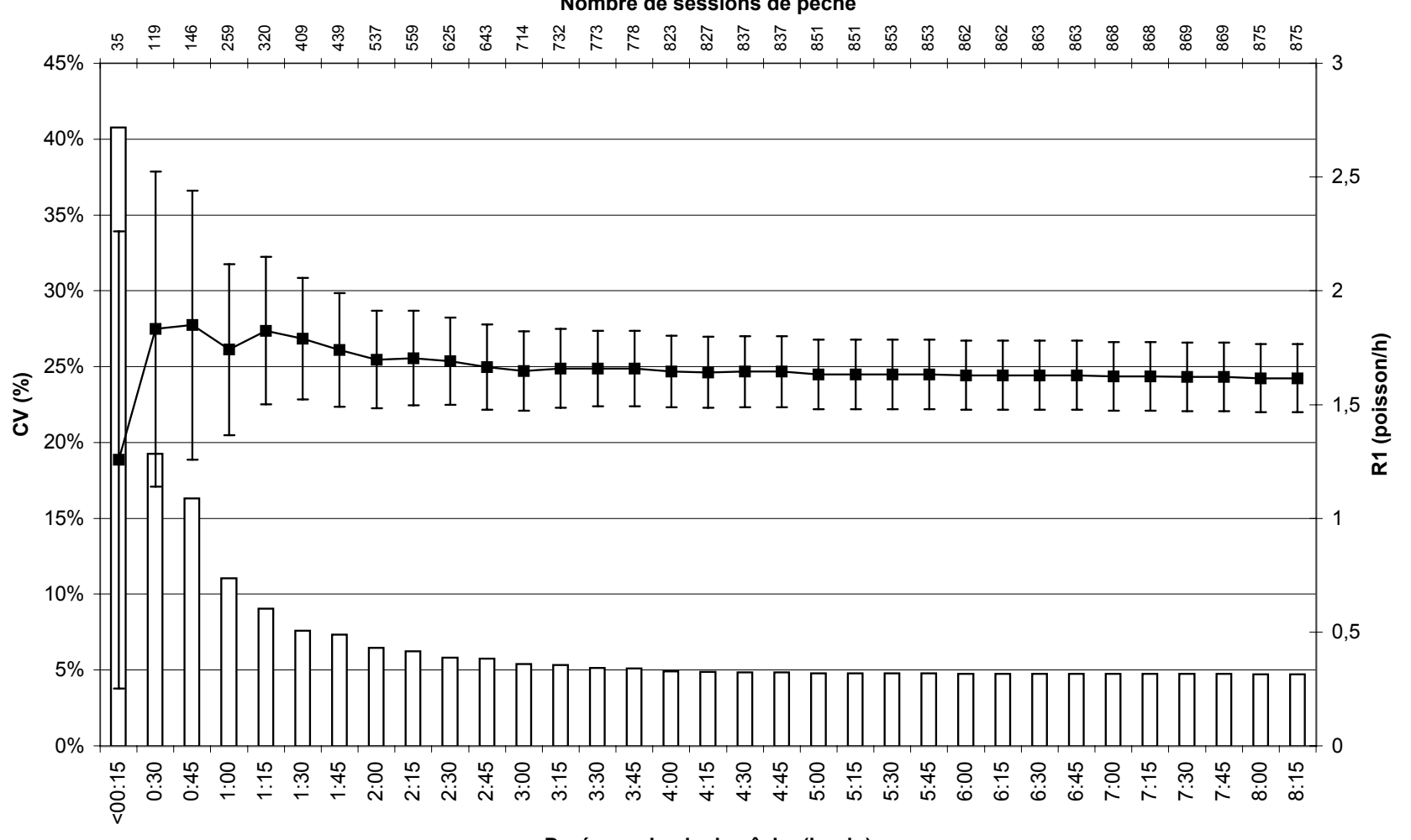

\section{Figure 1}

Durée maximale de pêche (h:min)

Estimation des captures de truites farios par unité d'effort au travers de la moyenne des ratios $\left(R_{1}\right.$ figurés par des points et des lignes avec précision de l'indice de confiance à $95 \%$ ) en fonction de la durée maximale de pêche prise en compte et du nombre de sessions. Evolution du coefficient de variation (CV figuré en histogramme).

\section{Figure 1}

Estimation of Brown Trout capture per unit of effort using the mean of the ratios $\left(R_{1}\right.$ represented by dots and line with $95 \%$ confidence interval) plotted against maximal session duration and number of fishing session included in the calculation. Evolution of the coefficient of variation (CV represented by bars). 


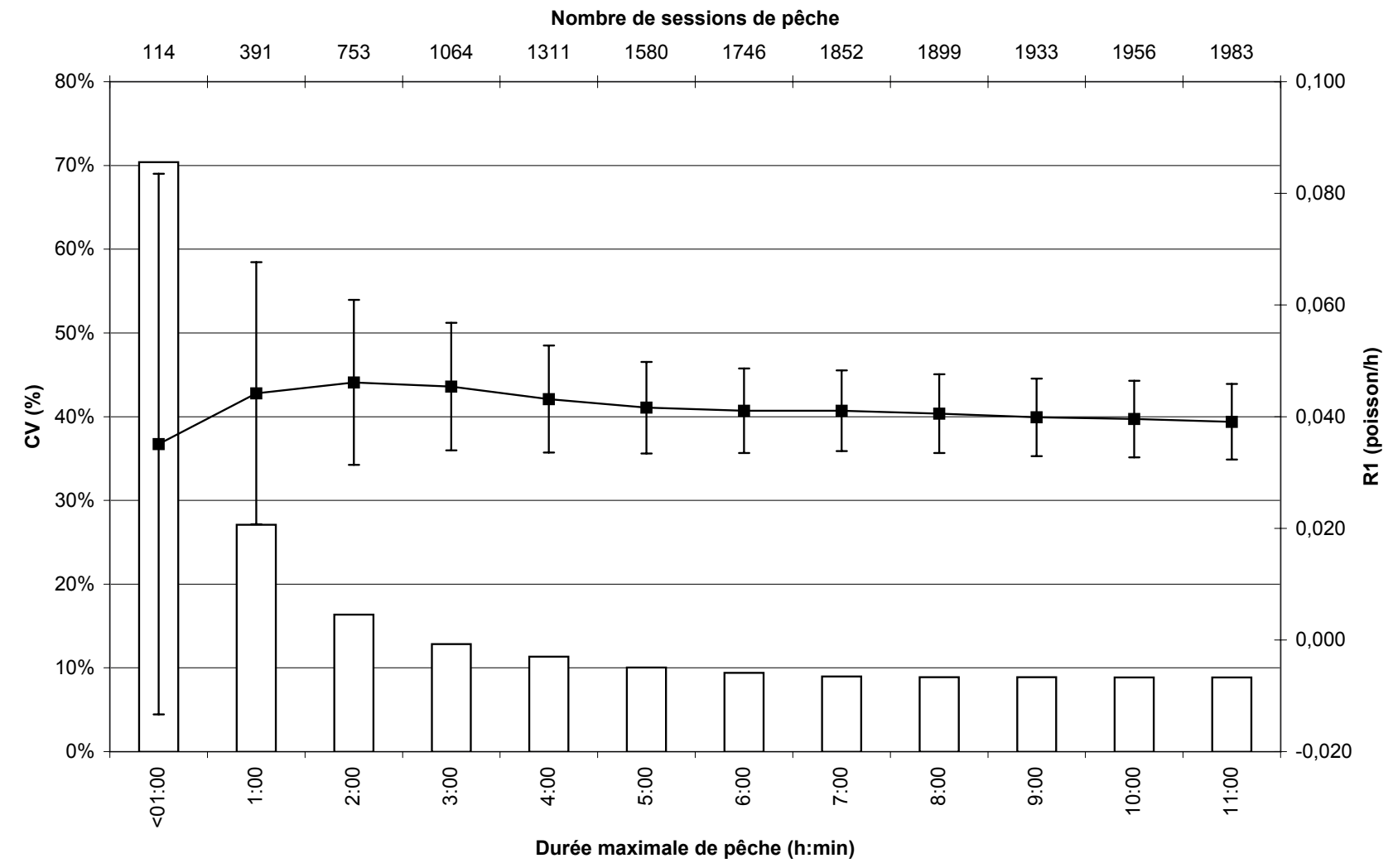

\section{Figure 2}

Estimation des captures de sandres par unité d'effort au travers de la moyenne des ratios ( $R_{1}$ figurés par des points et des lignes avec précision de l'indice de confiance à $95 \%$ ) en fonction de la durée maximale de pêche prise en compte et du nombre de sessions. Evolution du coefficient de variation (CV figuré en histogramme).

\section{Figure 2}

Estimation of Pikeperch capture per unit of effort using the mean of the ratios ( $R_{1}$ represented by dots and line with $95 \%$ confidence interval) plotted against maximal session duration and number of fishing session included in calculation. Evolution of the coefficient of variation (CV represented by bars). 


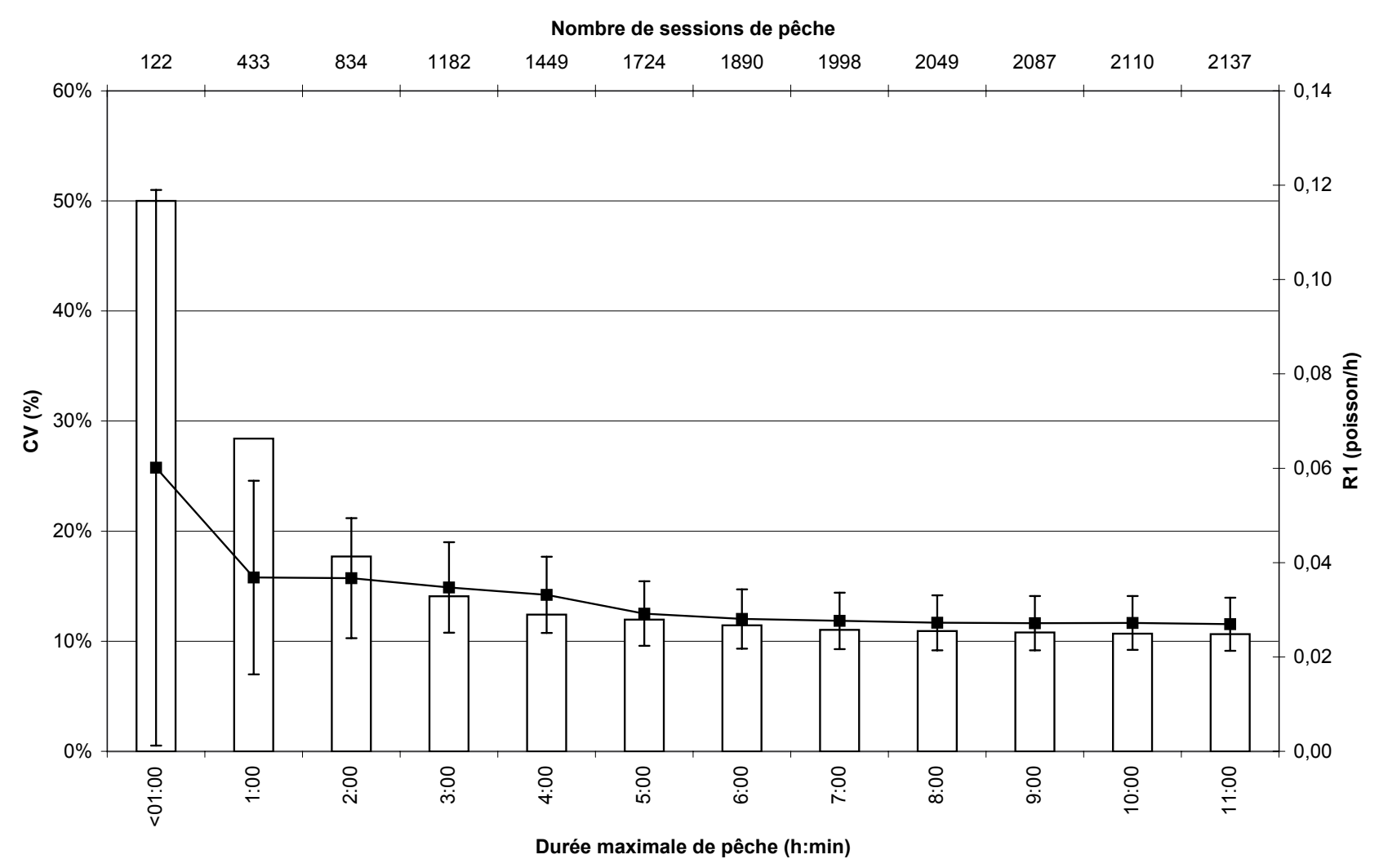

Figure 3

Estimation des captures de brochets par unité d'effort au travers de la moyenne des ratios $\left(R_{1}\right.$ figurés par des points et des lignes avec précision de l'indice de confiance à $95 \%$ ) en fonction de la durée maximale de pêche prise en compte et du nombre de sessions. Evolution du coefficient de variation (CV figuré en histogramme).

\section{Figure 3}

Estimation of Pike capture per unit of effort using the mean of the ratios $\left(R_{1}\right.$ represented by dots and line with $95 \%$ confidence interval) plotted against maximal session duration and number of fishing session included in calculation. Evolution of the coefficient of variation (CV represented by bars). 
Tous ces résultats sont le reflet de la densité des stocks sur les parcours échantillonnés : entre 1 et 10 poisson $/ 100 \mathrm{~m}^{2}$ pour les truites ; entre 1 et 10 poisson/ha, soit 100 fois moins, pour les brochets ou les sandres. La représentation importante de contextes salmonicoles conformes dans l'échantillon étudié explique les valeurs particulièrement élevées de CPUE truite. La sur représentation de contextes cyprinicoles dégradés conduit à des valeurs faibles de CPUE pour le brochet et le sandre.

La détection de différences significatives nécessite des échantillons plus importants lorsque le nombre poissons par heure est plus faible (WEITHMAN et HAVERLAND, 1991). Pour maintenir une précision constante, il est nécessaire d'augmenter l'échantillon lorsque le nombre de poissons par heure décroît (LESTER et al., 1991). II n'y a donc rien d'étonnant à ce que les valeurs de dispersions (CV et IC) soient plus élevées chez les carnassiers que chez la truite. II en découle que pour obtenir un effet équivalent sur l'estimateur, il faudra tronquer le calcul de manière différente suivant les espèces.

\section{Les profils}

Même si leur dispersion est telle qu'aucune différence significative n'est envisageable, les valeurs de l'estimateur présentent un profil différent. Les valeurs de CPUE calculées sur de très faibles durées sont inférieures à la valeur de convergence dans deux cas sur trois. Les petites valeurs observées pour la truite et le sandre peuvent s'interpréter comme un effet de mise en route de la pêche, durée pendant laquelle la probabilité de capture est plus faible.

En revanche, le brochet présente une valeur plus élevée pour les faibles durées de pêche qui laisse penser que, pour cette espèce, le début de pêche peut augmenter momentanément les chances de capture. Cela peut résulter de deux principaux facteurs : (1) le comportement particulier du poisson qui, contrairement au sandre, est un chasseur à l'affût, (2) l'emploi de techniques particulières présentant de meilleurs rendements pour le brochet.

Enfin, l'ajout des sessions de pêche les plus longues ne modifie pas beaucoup le résultat, ce qui laisse penser qu'une fois passés ces effets de mise en route de la pêche, les chances de captures sont constantes.

\section{Durée minimale à prendre en compte}

Nos résultats montrent que, même en incluant les CPUE calculées sur des durées de pêche très faibles, nous obtenons une précision correcte si (1) le nombre de sessions est important et (2) si les sessions de courtes durées sont peu nombreuses dans l'échantillon. Toutefois, si on réduit la taille de l'échantillon sans prêter attention à la durée minimale à prendre en compte, il existe bien un risque d'obtenir des CV importants.

La durée minimale à prendre en compte varie avec la précision recherchée dans les résultats. Les calculs présentés comprennent toujours les sessions à durées de pêche très faibles qui introduisent une variabilité supplémentaire. En les éliminant, les CV et les IC seront nécessairement meilleurs que ceux présentés Figures 1 à 3 . Si on fixe comme limite acceptable un $\mathrm{CV}$ de $30 \%$, on recommandera l'élimination des durées inférieures à 15 min pour la truite et à $1 \mathrm{~h}$ pour les carnassiers (brochet et sandre).

Pour la truite, qui présente des CPUE élevées, nous sommes en dessous des 30 minutes de durée minimale recommandées par HOENIG et al. (1997) et POLLOCK et al. (1997). Pour les carnassiers, qui présentent des CPUE faibles, nous sommes audessus. 


\section{CONCLUSION}

L'examen de la situation de trois espèces de poissons sur 14 sites différents montre que la durée minimale de pêche à prendre en compte augmente lorsque le rendement de pêche diminue. Dans les protocoles d'enquêtes itinérantes, nous préconisons de ne pas réduire cette durée en dessous de $15 \mathrm{~min}$ pour la truite et de $1 \mathrm{~h}$ pour les carnassiers (sandre et brochet). Cette durée peut également être retenue comme durée minimum d'interruption au delà de laquelle il est souhaitable de distinguer deux sessions de pêche pour un même pêcheur au cours d'une même journée (remarque applicable surtout aux carnets de pêche).

Par ailleurs, on sait qu'il est nécessaire d'augmenter le nombre de sessions à prendre en compte lorsque le rendement de pêche diminue (WEITHMAN et HAVERLAND, 1991 ; LESTER et al., 1991). Pour obtenir une précision relative de $20 \%$ sur les CPUE, nous préconisons un minimum de 178 sessions pour la truite, 1612 sessions pour le brochet et 1057 sessions pour le sandre (CHANGEUX et GALLET, 2002).

Un bon protocole doit donc faire le compromis entre le recueil d'un nombre suffisant de sessions et l'élimination d'un nombre limité de sessions trop courtes. Les durées minimum proposées conduisent dans tous les cas à l'élimination de moins de $10 \%$ des sessions. En revanche, en portant cette durée à 30 minutes pour la truite, on éliminera $119 / 875=13,6 \%$ des sessions, ce qui peut s'avérer problématique. Pour éviter cela, on organisera d'abord le passage de l'enquêteur de manière à éviter les débuts de session de pêche. Ensuite, il faudra considérer au cas par cas tous les facteurs susceptibles d'intervenir sur le rendement de pêche. Par exemple, un parcours à faible densité de poissons ou difficilement pêchable diminuera le rendement et il sera nécessaire non seulement d'augmenter la durée minimale de pêche par session, mais aussi d'augmenter le nombre de sessions. Enfin, il en sera de même si on souhaite estimer, non pas la CPUE, mais la récolte par unité d'effort (RPUE) qui est nécessairement plus faible car elle se limite aux poissons conservés. Dans ce cas, une taille légale en augmentation peut encore abaisser la RPUE et nécessiter également une augmentation de la durée minimale à prendre en compte. En revanche, lorsque la limitation des captures journalières par pêcheur est abaissée à des valeurs fréquemment atteintes (un carnassier par jour ou bien 2 à 3 truites par jour), la variabilité inter pêcheurs de la récolte sera réduite et pourra conduire à des RPUE plus précises malgré un nombre de sessions plus faible que celui préconisé.

\section{REMERCIEMENTS}

Ce travail a été réalisé grâce aux données rassemblées par la Cellule Nationale Expérimentation des Méthodes de Gestion du Conseil Supérieur de la Pêche. La collecte des données a été assurée par les brigades départementales de l'Ain, de l'Ariège, du Calvados, des Côtes d'Armor, du Finistère, de la Haute-Garonne, de l'llle-et-Vilaine, de l'Indre-et-Loire et des Deux-Sèvres avec la collaboration des personnels et des bénévoles des fédérations de pêche que nous remercions ainsi que Francis Chancerel et Françoise Bunch, qui nous ont donné accès à plusieurs références bibliographiques. 


\section{BIBLIOGRAPHIE}

CHANGEUX T., GALLET O., 2002. Protocole pour le suivi national de la pêche aux lignes en 2002. Note du service technique, Conseil Supérieur de la Pêche, Direction Générale, Paris (France). 8 p. +6 annexes.

CHANGEUX T., BONNIEUX F., ARMAND C., 2001. Cost benefit analysis of fisheries management plans. Fisheries Management and Ecology, 8, 425-434.

DAGNELI P., 1992. Statistiques théoriques et appliquées, tome 1. Presses agronomiques de Gembloux, Gembloux (Belgique), 492 p.

FATORA J.R., 1974. Noontoola - a sixteen year creel and use history of a southern appalachian trout stream under changing management regulation. In : WEBB J.W. (Eds.), Proceedings of the $24^{\text {th }}$ annual conference of southeastern association of game and fish commissioners, 622-637, Atlanta, Georgie (Etats Unis).

GALLET O., 2001. Premiers éléments d'évaluation de la pratique de la pêche et de l'état des contextes à l'aide des déclarations de captures par les pêcheurs amateurs aux lignes. Rapport de la Cellule nationale de développement et de vulgarisation des méthodes de gestion piscicoles, Conseil Supérieur de la Pêche, Délégation Régionale de Rennes (France). 13 p. + 2 annexes.

GERARD P., 1993. Communauté piscicole de deux canaux et évaluation de l'impact de la pêche récréative sur la dynamique du gardon Rutilus rutilus (L.). Thèse de doctorat, Université de Louvain (Belgique), $196 \mathrm{p}$.

HANSEN J.H., BEARD T.D. Jr., HEWETT S.W., 2000. Catch rates and catchability of walleyes in angling and spearing fisheries in northern Wisconsin lakes. North American Journal of Fisheries Management, 20, 109-118.

HOENIG J.M., JONES C.M., POLLOCK K.H., ROBSON D.S., WADE D.L., 1997. Calculation of catch rate and total catch in roving surveys of anglers. Biometrics, 53, 306-317.

KEMPINGER J.J., CARLINE R.F., 1978. Change in population density, growth and harvest of northern pike in Escanaba lake. Technical Bulletin, 104, Department of natural resources, Madison, Wisconsin, $17 \mathrm{p}$.

LATTA W.C., 1972. The northern pike in Michigan: a simulation of regulation for fishing. Michigan Academician, 5, 153-169.

LESTER N.P., PETZOLD M.M., DUNLOP W.I., 1991. Sample size determination in roving creel surveys. In : GUTHERIE D., HOENIG J.M., HOLLIDAY M., JONES C., MILLS M.J., MOBERLY S.A., POLLOCK K.H., TALHELM D.R. (Eds.), Creel and anglers surveys in fisheries management. American Fisheries Society, Symposium 12, Bethesda, Maryland (Etats Unis), 25-39.

MACKENZIE C., 1991. Comparison of northern pike catch and harvest rates estimated from uncompleted and completed fishing trips. In : GUTHERIE D., HOENIG J.M., HOLLIDAY M., JONES C., MILLS M.J., MOBERLY S.A., POLLOCK K.H., TALHELM D.R. (Eds.), Creel and anglers surveys in fisheries management. American Fisheries Society, Symposium 12, Bethesda, Maryland (Etats Unis), 47-50.

MALVESTUTO S.P., 1996. Sampling the recreational creel. In : MURPHY B.R., WILLIS W.W. (Eds.), Fisheries Techniques, $2^{\text {nd }}$ Edition, American Fisheries Society, Bethesda, Maryland (Etats Unis), 591-623.

POLLOCK K.H., JONES C.M., BROWN T.L., 1994. Angler survey methods and their application in fisheries management. American Fisheries Society Special Publication $\mathrm{n}^{\circ} 12$, Bethesda, Maryland (Etats Unis), $371 \mathrm{p}$.

POLLOCK K.H, HOENIG J.M., JONES C.M., ROBSON D.S., GREEN C.J., 1997. Catch rate estimation for roving and access point surveys. North American Journal of Fisheries Management, 17, 11-19.

WEITHMAN A.S., HAVERLAND P., 1991. Comparability of data collected by telephone and roving creel surveys. In : GUTHERIE D., HOENIG J.M., HOLLIDAY M., JONES C., MILLS M.J., MOBERLY S.A., POLLOCK K.H., TALHELM D.R. (Eds.), Creel and anglers surveys in fisheries management. American Fisheries Society, Symposium 12, Bethesda, Maryland (Etats Unis), 67-73. 
